\title{
The existence and uniqueness theorems of fuzzy delay differential equations
}

\author{
Nor Atirah Izzah Zulkefli*, Normah Maan \\ Department of Mathematics, Faculty of Science, UTM, 81310 UTM Skudai, Johor, Malaysia \\ *Corresponding Author: noratirahizzah@gmail.com (N.A.I. Zulkefli)
}

\section{Article history :}

Received 15 February 2014

Revised 11 April 2014

Accepted 7 June 2014

Available online 5 July 2014

GRAPHICAL ABSTRACT

\begin{abstract}
Delay differential equations (DDEs) arise many different phenomena including in physics, biology and chemistry. In many cases of the modeling of real world problems, information about the behaviour of a dynamical system is uncertain. In order to obtain a more realistic model, we have to take into account these uncertainties. Therefore, in this paper, we propose the existence and uniqueness theorems for fuzzy timedelay dynamical systems. We finally present some conclusions and new directions for further research in this area.
\end{abstract}

Keywords: Fuzzy Delay Differential Equation (FDDE), Fuzzy Time-Delay Dynamical Systems, Existence and Uniqueness Theorems

\section{INTRODUCTION}

Delay differential equations (DDEs) are differential equations in which the derivative of the unknown function at certain time is given in term of the value of the function at previous times. These types of equations are a large and important class of dynamical systems. They often arise in either natural or technological control problems. The delay may appear because of physical properties of equipment used in the system, signal transmission or measurement of system variables. For example, actuators, sensors and field networks which are involved in feedback loops may exhibit delays. Time-delay systems are also used to model several different mechanisms in the dynamics of epidemics [1].

In many cases of the modelling of real world phenomena, information about the behaviour of a dynamical system is uncertain. In order to obtain a more realistic model, we have to take into account these uncertainties. Fuzzy differential equations are a natural way to model dynamical systems under uncertainty. This type of system will provide a better representation of the real world problems. Therefore the study of this topic has been rapidly growing in recent years. It was first started by Chang and Zadeh [2], who introduced the concept of fuzzy derivative. It was followed up by Dubois and Prade [3] in 1982. They used the extension principle in their approach. In 1980, Kandel and Byatt [4] applied the concept of fuzzy differential equation to the analysis of fuzzy dynamical problems. The researches have been continuing unlimited to the theory of fuzzy differential equations but also the application in the real problems as were reported in many literatures $[5,6,7,8,9]$. The existence and uniqueness theorem is one of the most important and fundamental theorems in the theory of classical differential equation. The theorems that deal with fuzzy-set function were also discussed in many literatures. Some of them can be found in $[10,11,12]$. Xiaowei and Zhongfeng [10] proved a new existence and uniqueness theorem for fuzzy differential equation. This theorem was different from previous works since, they use Liu process [10]. Balachandran and Prakash [11] proved the solutions of fuzzy delay differential equations with nonlocal condition. In [12], Lupulescu and Abbas proved a local existence and uniqueness result for fuzzy delay differential equations driven by Liu process.

Therefore, in this paper we will derive the existence and uniqueness theorems of a specific fuzzy delay differential equation (FDDE). This paper will focus on the existence and uniqueness theorems of fuzzy time-delay dynamical systems. The organization of this paper is as follows. In Section 2, some notations, concepts and the basic definitions of delay differential equations are briefly presented. In Section 3, introduces fuzzy delay differential equations and the existence and uniqueness theorems for fuzzy time-delay dynamical systems. Finally, Section 4 presents concluding remarks.

\section{PRELIMINARIES}

Definition 1: [13] If $x$ is a function defined at least on $[t-r, t] \rightarrow \mathfrak{R}^{n}$ then we define a new function $x_{t}$ : $[-r, 0] \rightarrow \mathfrak{R}^{n}$ by 


$$
x_{t}(\theta)=x(t+\theta),-r \leq \theta \leq 0 .
$$

If $x$ is continuous on $[t-r, t] \rightarrow D \subset \Re$, then $x_{t}$ is continuous on $[-r, 0]$, i.e. $x_{t} \in \zeta_{D}$, where $r>0$, we denote by $\zeta=C\left([-r, 0] \rightarrow \mathfrak{R}^{n}\right)$. Let $\phi \in \zeta$. We will take the norm on this space to be $\|\phi\|_{r}=\sup _{-r \leq \theta \leq 0}\|\phi(\theta)\|$, where $\|$.$\| is the usual Euclidean norm on \mathfrak{R}^{n}$. With this norm, $\zeta$ is a Banach space. Further, for $D \subseteq \mathfrak{R}^{n}$ let $\zeta_{D}=$ $C([-r, 0] \rightarrow D)$ be the set of continuous fuzzy functions $[-r, 0]$ into $D$.

In the following, unless otherwise stated, we will take $J \subseteq$ $\mathfrak{R}$ and $D \subseteq \mathfrak{R}^{n}$ to be open sets.

Definition 2: [13] If $F: J \times \zeta_{D} \rightarrow \mathfrak{R}^{n}$ is a given fuzzy functional and ' represent the right hand derivative, we call the relation

$$
x^{\prime}(t)=F\left(t, X_{t}\right)
$$

a delay differential equation on $J \times \zeta_{D}$.

Definition 3: [13] Let $F: J \times \zeta_{D} \rightarrow \Re^{n}$. A function $x(t)$ is said to be a solution of (2) on $\left[t_{0}-r, \beta\right)$ if there are $t_{0} \in$ $\mathfrak{R}^{n}$ and $\beta>t_{0}$ such that

$$
\begin{array}{cl}
\text { i. } & x \in C\left(\left[t_{0}-r, \beta\right), D\right), \\
\text { ii. } & {\left[t_{0}, \beta\right) \subset J,} \\
\text { iii. } & x(t) \text { satisfies (2) for } t \in\left[t_{0}, \beta\right) .
\end{array}
$$

For a given $t_{0} \in \mathfrak{R}$ and $\phi \in \zeta_{D}$, the initial value problem associated with the FDDE (2) is

$$
\begin{aligned}
& x^{\prime}(t)=F\left(t, x_{t}\right), \quad t \geq t_{0}, \\
& x_{t_{0}}=\phi_{0}, \\
& \text { or } \quad t \geq t_{0}, \\
& x^{\prime}(t)=F\left(t, x_{t}\right), \quad t \\
& x_{t_{0}}=\phi_{0}\left(t-t_{0}\right) .
\end{aligned}
$$

Continuity Condition (C) [6] is satisfied if $F\left(t, x_{t}\right)$ is continuous with respect to $t$ in $\left[t_{0}, \beta\right]$ for each given continuous function $x:\left[t_{0}-r, \beta\right) \rightarrow D$.

If $F$ satisfies Condition (C) then a continuous function $x:\left[t_{0}-r, \beta\right) \rightarrow D$ is a solution of Equation (4) if and only if

$x(t)=\left\{\begin{array}{cr}\phi\left(t-t_{0}\right), & {\left[t_{0}-r, t_{0}\right]} \\ \phi(0)+\int_{t_{0}}^{t} \hat{F}\left(s, x_{s}\right) d s, & {\left[t_{0} \leq T\right]}\end{array}\right.$

Definition 4: [13] The function $x(t)$ is a solution of the initial value problem (4) on $\left[t_{0}-r, \beta\right)$ if $x(t)$ is a solution of (2) on $\left[t_{0}-r, \beta\right)$ and $x_{t_{0}}=\phi_{0}$.

Lemma 1: [13] If $x$ is continuous on $\left[t_{0}-r, t_{0}+\gamma\right]$, then $x_{t}$ is a continuous function of $t$ for $t \in\left[t_{0}, t_{0}+\gamma\right]$.

\section{FUZZY DELAY DIFFERENTIAL EQUATION}

Consider the first-order fuzzy time-delay initial value differential equation given by

$$
\begin{aligned}
& x^{\prime}(t)=F x(t)+G x(t-r), \quad t \in\left[t_{0}, T\right] \\
& x(t)=x_{0}, \quad t \in\left[t_{0}-r, t_{0}\right]
\end{aligned}
$$

where $\quad x(t)$ and $(t-r) \in\{x(\tau): \tau<t\}$ are $n-$ dimensional fuzzy functions of $t$, every element of matrices $A=\left[a_{i j}\right]_{n \times n}, a_{i j} \in F(\Re)$ and $\quad B=\left[b_{i j}\right]_{n \times n}, b_{i j} \in$ $F(\Re)$ are supposed to be fuzzy numbers where $F(\mathfrak{R})$ represents the fuzzy sets defined on $\mathfrak{R}$. The function $x^{\prime}(t)$ is the fuzzy derivative of $x(t)$ at $t \in I$ and $x_{0}$ is a fuzzy number and the time-delay $r$ is a known positive rational number.

Most of these steps follow from [13].

Definition 5: If $x$ is a function defined at least on $[t-r, t] \rightarrow \mathfrak{R}$ then we define a new function $x_{t}$ : $[-r, 0] \rightarrow \mathfrak{R}$ by

$$
x_{t}(\theta)=x(t+\theta),-r \leq \theta \leq 0 \text {. }
$$

If $x$ is continuous on $[t-r, t] \rightarrow D \subset \Re$, then $x_{t}$ is continuous on $[-r, 0]$, i.e. $x_{t} \in \zeta_{D}$, where $r>0$, we denote by $\zeta=C([-r, 0] \rightarrow \Re)$. Let $\phi \in \zeta$. We will take the norm on this space to be ||$\phi||_{r}=\sup _{-r \leq \theta \leq 0}\|\phi(\theta)\|$, where $\|$.$\| is the usual Euclidean norm on \mathfrak{R}$. Further, for $D \subseteq \Re$ let $\zeta_{D}=C([-r, 0] \rightarrow D)$ be the set of continuous fuzzy functions $[-r, 0]$ into $D$.

In the following, unless otherwise stated, we will take $J \subseteq$ $\mathfrak{R}$ and $D \subseteq \Re$ to be open sets.

Definition 6: If $\hat{F}: J \times \zeta_{D} \rightarrow \Re$ is a given fuzzy functional and ' represent the right hand derivative, we call the relation

$$
x^{\prime}(t)=\hat{F}\left(t, X_{t}\right)
$$

a fuzzy delay differential equation on $J \times \zeta_{D}$.

Definition 7: Let $\widehat{F}: J \times \zeta_{D} \rightarrow \mathfrak{R}$. A function $x(t)$ is said to be a solution of (8) on $\left[t_{0}-r, \beta\right)$ if there are $t_{0} \in \Re$ and $\beta>t_{0}$ such that

$$
\begin{aligned}
\text { i. } & x \in C\left(\left[t_{0}-r, \beta\right), D\right), \\
\text { ii. } & {\left[t_{0}, \beta\right) \subset J, } \\
\text { iii. } & x(t) \operatorname{satisfies~}(7) \text { for } t \in\left[t_{0}, \beta\right) .
\end{aligned}
$$

For a given $t_{0} \in \mathfrak{R}$ and $\phi \in \zeta_{D}$, the initial value problem associated with the FDDE (8) is

$$
\begin{aligned}
x^{\prime}(t) & =\hat{F}\left(t, x_{t}\right), \quad t \geq t_{0}, \\
x_{t_{0}} & =\phi_{0},
\end{aligned}
$$


or

$$
\begin{aligned}
x^{\prime}(t) & =\hat{F}\left(t, x_{t}\right), \quad t \geq t_{0}, \\
x_{t_{0}} & =\phi_{0}\left(t-t_{0}\right) .
\end{aligned}
$$

Continuity Condition (C) is satisfied if $\hat{F}\left(t, x_{t}\right)$ is continuous with respect to $t$ in $\left[t_{0}, \beta\right]$ for each given continuous function $x:\left[t_{0}-r, \beta\right) \rightarrow D$.

If $\hat{F}$ satisfies Condition (C) then a continuous function $x:\left[t_{0}-r, \beta\right) \rightarrow D$ is a solution of Equation (10) if and only if

$x(t)=\left\{\begin{array}{cr}\phi\left(t-t_{0}\right), & {\left[t_{0}-r, t_{0}\right]} \\ \phi(0)+\int_{t_{0}}^{t} \hat{F}\left(s, x_{s}\right) d s, & {\left[t_{0} \leq T\right]}\end{array}\right.$

Definition 8: The function $x(t)$ is a solution of the initial value problem (10) on $\left[t_{0}-r, \beta\right)$ if $x(t)$ is a solution of (7) on $\left[t_{0}-r, \beta\right)$ and $x_{t_{0}}=\phi_{0}$.

Definition 9: Let $\hat{F}: J \times \zeta_{D} \rightarrow \mathfrak{R}^{n}$ and let $\xi \subset J \times \zeta_{D}$. We say that $\hat{F}$ is Lipschitz on $\xi$ if there exists $K \geq 0$ such that

$$
\hat{F}(t, \psi)-\hat{F}(t, \bar{\psi}) \leq K\|\psi-\bar{\psi}\|_{r}
$$

whenever $(t, \psi)$ and $(t, \bar{\psi}) \in \xi$.

Definition 10: The functional $\hat{F}: J \times \zeta_{D} \rightarrow \mathfrak{R}$ is said to be locally Lipschitz if for each given $(\bar{t}, \bar{\psi}) \in J \times \zeta_{D}$ there exist numbers $a>0$ and $b>0$ such that

$$
\xi \equiv(\overline{[t}-a, \bar{t}+a] \cap J) \times\left\{\psi \in \zeta:\|\psi-\bar{\psi}\|_{r} \leq b\right\}
$$

is a subset of $J \times \zeta_{D}$ and $\hat{F}$ is Lipschitz on $\xi$. (The Lipschitz constant for $F$ and $G$ depends, in general, on the particular set $\xi$ ).

\subsection{Existence and Uniqueness Theorems for Fuzzy Time-Delay Dynamical Systems}

Theorem 1: (Existence) Let $\hat{F}:\left[t_{0}, \alpha\right) \times \zeta_{D} \rightarrow \mathfrak{R}$ be continuous and locally Lipschitz. Then for each $\phi \in \zeta_{D}$, there exists $T>t$ such that the fuzzy time-delay initial value problem (6) has a unique solution on $\left[t_{0}-r, t_{0}+\Delta\right.$ ) for some $\Delta>0$.

Proof Choose any $a>0$ and $b>0$ sufficiently small so that

$$
\xi \equiv\left[t_{0}, t_{0}+a\right] \times\left\{\psi \in \zeta:\left\|\psi-\phi_{0}\right\|_{r} \leq b\right\}
$$

is a subset of $\left[t_{0}, \alpha\right) \times \zeta_{D}$ and $\hat{F}$ is Lipschitz on $\xi$ with Lipschitz constant $K$. Define a continuous function $\bar{\chi}$ on $\left[t_{0}=r, t_{0}+a\right]$,

$$
\bar{\chi}(t)=\left\{\begin{aligned}
\phi_{0}\left(t-t_{0}\right), & t_{0}-r \leq t \leq t_{0} \\
\phi_{0}(0), & t_{0} \leq t \leq t_{0}+a .
\end{aligned}\right.
$$

Then $\hat{F}\left(t, \bar{\chi}_{t}\right)$ depends continuously on $t$, and hence $\left\|\hat{F}\left(t, \bar{\chi}_{t}\right)\right\| \leq B_{1}$ on $\left[t_{0}, t_{0}+a\right]$ for some constant $B_{1}$.

Now define $B=K b+B_{1}$. Choose $a_{1} \in(0, a]$ such that

$$
|| \bar{\chi}_{t}-\phi||_{r}=|| \bar{\chi}_{t}-\bar{\chi}_{t_{0}} \| \leq b \text { for } t_{0} \leq t_{0}+a_{1}
$$

Choose $\Delta>0$ such that

$$
\Delta \leq \min \left\{a_{1}, b \backslash B\right\} \text { and } \Delta<a \backslash K \text {. }
$$

Let $S$ be the set of all continuous functions $\chi:\left[t_{0}-r, t_{0}+\right.$ $\Delta] \rightarrow \mathfrak{R}$ such that

$\chi(t)=\phi\left(t-t_{0}\right), \quad$ for $t_{0}-r \leq t \leq t_{0}$, and

||$\chi(t)-\phi(0) \| \leq b$, for $t_{0} \leq t \leq t_{0}+\Delta$.

Note that if $\chi \in S$ and $\in\left[t_{0}, t_{0}+\Delta\right]$, then $\left\|\chi_{t}-\bar{\chi}_{t}\right\|$, so that

$$
\begin{aligned}
\left\|F\left(t, \chi_{t}\right)\right\|+\left\|G\left(t, \chi_{t}\right)\right\| & \leq K\left\|\chi_{t}-\bar{\chi}_{t}\right\|_{r}+B_{1} \\
& \leq B .
\end{aligned}
$$

For each $\chi \in S$ define a function $T \chi$ on $\left[t_{0}-r, t_{0}+\Delta\right]$ by

$$
\begin{aligned}
& T \chi(t) \\
& =\left\{\begin{array}{lr}
\phi_{0}\left(t-t_{0}\right), & t_{0}-r \leq t \leq t_{0}, \\
\phi_{0}(0)+\int_{t_{0}}^{t} \hat{F}\left(s, \chi_{s}\right) d s, & t_{0} \leq t \leq t_{0}+\Delta .
\end{array}\right.
\end{aligned}
$$

Then $t \chi$ is continuous and, since $\left\|\hat{F}\left(s, \chi_{s}\right)\right\| \leq B$,

$$
\|(T \chi)(t)-\phi(0)\| \leq B \Delta \leq b \text { for } t_{0} \leq t \leq t_{0}+\Delta .
$$

Choose $x_{(0)} \in S$ and construct the successive approximations $x_{(1)}=T x_{(0)}, x_{(2)}=T x_{(1)}, \ldots$

Note that for each $\ell, x_{(\ell)}(t)=\phi\left(t-t_{0}\right)$ on $\left[t_{0}-r, t_{0}\right]$. We will now prove that the sequence $\left\{x_{(\ell)}(t)\right\}$ converges. For each $\ell=0,1,2, \ldots$ then $t_{0} \leq t \leq t_{0}+\Delta$,

$$
\begin{aligned}
& \left\|x_{(\ell+2)}(t)-x_{(\ell+1)}(t)\right\| \\
& =\left\|\int_{t_{0}}^{t}\left[\hat{F}\left(s, x_{(\ell+1) s}\right)-\hat{F}\left(s, x_{(\ell) s}\right)\right] d s\right\| \\
& \quad \leq K \Delta \sup _{t_{0} \leq s \leq t_{0}+\Delta}\left\|x_{(\ell+1) s}-x_{(\ell) s}\right\| .
\end{aligned}
$$

Note that $\left\|x_{1}(t)-x_{0}(t)\right\| \leq 2 b$ on $\left[t_{0}-r, t_{0}+\Delta\right]$. Thus $\left\|x_{(1) t}-x_{(0) t}\right\| \leq 2 b$ on $\left[t_{0}, t_{0}+\Delta\right]$ and

$$
\begin{aligned}
\left\|x_{2}(t)-x_{1}(t)\right\| & \leq \int_{t_{0}}^{t} K\left\|x_{(1) s}-x_{(0) s}\right\| d s \\
& \leq 2 b K\left(t-t_{0}\right)
\end{aligned}
$$


on $\left[t_{0}, t_{0}+\Delta\right]$ which further implies $\left\|x_{(2) t}-x_{(1) t}\right\|_{r} \leq$ $2 b K\left(t-t_{0}\right)$ on $\left[t_{0}, t_{0}+\Delta\right]$. This leads to

$$
\begin{aligned}
\left\|x_{3}(t)-x_{2}(t)\right\| & \leq \int_{t_{0}}^{t} K\left\|x_{(2) s}-x_{(1) s}\right\| d s \\
& \leq 2 b \frac{K^{2}\left(t-t_{0}\right)^{2}}{2}
\end{aligned}
$$

and, by induction,

$$
\left\|x_{(\ell+1)}(t)-x_{(\ell)}(t)\right\| \leq 2 b \frac{K^{\ell}\left(t-t_{0}\right)^{\ell}}{\ell !}
$$

on $\left[t_{0}, t_{0}+\Delta\right]$. This, together with $x_{(\ell+1)}(t)=x_{(\ell)}(t)$ on $\left[t_{0}-r, t_{0}\right]$ gives

$$
\left\|x_{(\ell+1)}(t)-x_{(\ell)}(t)\right\| \leq 2 b \frac{K^{\ell}(\Delta)^{\ell}}{\ell !}
$$

on $\left[t_{0}-r, t_{0}+\Delta\right]$. Now, the series

$$
x_{(0)}(t)+\sum_{p=0}^{\infty}\left[x_{(p+1)}(t)-x_{(p)}(t)\right]
$$

converges, the convergence of the sequence $\left\{x_{(\ell)}\right\}$ follows by application of the comparison test of each component of

$x_{(\ell)}(t)=x_{(0)}(t)+\sum_{p=0}^{\ell-1}\left[x_{(p+1)}(t)-x_{(p)}(t)\right]$

on $\left[t_{0}-r, t_{0}+\Delta\right]$.

Let $x(t) \equiv \lim _{\ell \rightarrow \infty} x_{(\ell)}(t)$ for $t_{0} \leq t \leq t_{0}+\Delta$. Clearly $x(t)$ is continuous on $\left[t_{0}-r, t_{0}+\Delta\right]$ and $x_{t_{0}}=\phi_{0}$. Further $\left.\left\|x(t)-x_{(\ell)}(t)\right\| \leq 2 b \sum_{p=\ell}^{\infty} \frac{K^{p}(\Delta)^{p}}{p !}\right]$ for $t_{0}-r \leq t \leq t_{0}+$ $\Delta$ and $\left.\left\|x_{t}-x_{(\ell) t}\right\|_{r} \leq 2 b \sum_{p=\ell}^{\infty} \frac{K^{p}(\Delta)^{p}}{p !}\right]$ for $t_{0} \leq t \leq t_{0}+$ $\Delta$. Thus, $t_{0} \leq t \leq t_{0}+\Delta$ for any $\ell=0,1,2, \ldots$

$$
\begin{aligned}
\| x(t)- & \phi_{0}(0)-\left[\int_{t_{0}}^{t} \hat{F}\left(s, x_{s}\right) d s\right]\|\leq\| x(t)-x_{(\ell)}(t) \| \\
& +\left\|\int_{t_{0}}^{t} \hat{F}\left(s, x_{(\ell-1) s}\right)-\hat{F}\left(s, x_{s}\right) d s\right\| \\
& \leq 2 b \sum_{p=\ell}^{\infty} \frac{K^{p}(\Delta)^{p}}{p !}+K \Delta 2 b \sum_{p=\ell-1}^{\infty} \frac{K^{p}(\Delta)^{p}}{p !} .
\end{aligned}
$$

Taking the limit as $\ell \rightarrow \infty$ of this inequality then gives

$$
\left\|x(t)-\phi_{0}(0)-\left[\int_{t_{0}}^{t} \hat{F}\left(s, x_{s}\right) d s\right]\right\|=0 .
$$

Since the right hand side tend to 0 , it follows that the left hand side must be 0 . Hence $x(t)$ satisfies (11) which means that $x(t)$ is a solution of (6).

Theorem 2: (Uniqueness) Let $\hat{F}:\left[t_{0}, \alpha\right) \times \zeta_{D} \rightarrow \Re$ be continuous and locally Lipschitz on its domain. Then given any $\phi \in \zeta_{D}$, there is at most one solution of the initial value problem (6) on $\left[t_{0}-r, \beta\right)$ for any $\beta \in\left(t_{0}, \alpha\right]$.
Proof Suppose (for contradiction) that for some $\beta \in\left(t_{0}, \alpha\right]$ there are two solutions $x$ and $\tilde{x}$ mapping $\left[t_{0}-r, \beta\right) \rightarrow D$ with $x \neq \tilde{x}$. Let

$$
t_{1}=\inf \left\{t \in\left(t_{0}, \beta\right): x(t) \neq \tilde{x}(t)\right\}
$$

Then $t_{0} \leq t_{1}<\beta$ and

$$
x(t)=\tilde{x}(t) \text { for } t_{0}-r \leq t_{0}<t_{1} .
$$

Since $\left(t_{1}, x_{t_{1}}\right) \in\left[t_{0}, \beta\right) \times \zeta_{D}$, there exist numbers $a>0$ and $b>0$ such that the set

$$
\xi \equiv\left[t_{1}, t_{1}+a\right] \times\left\{\psi \in \zeta:\left\|\psi-x_{t_{1}}\right\|_{r} \leq b\right\}
$$

is contained in $\left[t_{0}, \alpha\right) \times \zeta_{D}$ and $\hat{F}$ is Lipschitz on $\xi$ (with Lipschitz constant $K$ ).

By Lemma 1, there exists $\delta \in(0, a]$ such that $\left(t, x_{t}\right) \in \xi$ and $\left(t, \tilde{x}_{t}\right) \in \xi$ for $t_{1} \leq t<t_{1}+\delta$. Thus, for $t_{1} \leq t<$ $t_{1}+\delta$,

$$
\begin{aligned}
\|x(t)-\tilde{x}\| & =\left\|\int_{t_{0}}^{t}\left[\hat{F}\left(s, x_{s}\right)-\hat{F}\left(s, \tilde{x}_{s}\right)\right] d s\right\| \\
& \leq \int_{t_{1}}^{t} K\left\|x_{s}-\tilde{x}_{s}\right\|_{r} d s .
\end{aligned}
$$

Define $v(s)=\left\|x_{s}-\tilde{x}_{s}\right\|_{r}$ then

$$
\|x(t)-\tilde{x}\| \leq \int_{t_{1}}^{t} K v(s)_{r} d s .
$$

From this and the Reid's Lemma it follows that $v(t)=0$, and hence $x(t)=\tilde{x}(t)$ on $\left[t_{1}, t_{1}+\delta\right)$, contradicting the definition of $t_{1}$. Since $x(t)=\tilde{x}(t)$, therefore there cannot be two different solutions on its domain.

\section{CONCLUSION}

In this paper we introduced specific fuzzy delay differential equation which is fuzzy time-delay dynamical systems. By Banach Space, we proved an existence and uniqueness theorem for fuzzy time-delay dynamical systems under Lipschitz condition. For further research, numerial solution and stability analysis on this system will be considered.

\section{ACKNOWLEDGEMENT}

This research is supported by Research Management Centre - UTM and Ministry of Higher Education (MOHE) Grants through votes 4F127 and 07J77. The authors are thankful for the financial support.

\section{REFERENCES}

[1] J. Koshkouei and M. H. Farahi and K. J. Burnham, International Journal of Control, (to appear), 85 (2012) 147.

[2] S. L. Chang and L.A. Zadeh, IEEE Transactions on Systems, Man 
and Cybernetics, 2 (1972) 330.

[3] D. Dubois and H. Prade, Fuzzy Sets and Systems, 8 (1982) 225.

[4] A. Kandel and W. J. Byatt, Proceedings of the International

Conference on Cybernetics and Society Tokyo, (1978) 1213

[5] B. Bede and I. J. Rudas and A. L. Bencsik, Information Sciences, 177 (2007) 1648.

[6] T. Jayakumar and D. Maheskumar and K. Kanagarajan, Applied Mathemtical Sciences, 6 (2012) 2989.

[7] S. Song and Congxin Wu, Information Sciences, 108 (1998) 123.

[8] J. Buckley and L. J. Jowers, Simulating Continuous Fuzzy Systems, (2006) 188.
[9] L. J. Jowers and J. Buckley and K. D. Reilly, Information Sciences, 177 (2007) 436.

[10] Xiaowei and Q. Zhongfeng, International Journal of Fuzzy Systems, 13 (2011) 148.

[11] K. Balachandran and P. Prakash, Journal of Korean Society for Industrial and Applied Mathematics, 6 (2002) 81.

[12] V. Lupulescu and U. Abbas, Fuzzy Optim Decis Making, 11 (2012) 99.

[13] R. D. Driver, Existence and Stability of Solutions of a DelayDifferential System, Arch. Rational Mech. Anal., (1962). 